\title{
Which Galaxy Property Best Predicts Quiescence?
}

\author{
Joel Leja ${ }^{1}$, Pieter van Dokkum ${ }^{1}$ and the 3D-HST Collaboration \\ ${ }^{1}$ Department of Astronomy, Yale University, New Haven, CT 06520-8101 \\ email: joel.leja@yale.edu \\ email: pieter.vandokkum@yale.edu
}

\begin{abstract}
It is generally accepted that local elliptical galaxies assembled most of their mass in a burst of star formation between $1<z<3$, yet today, their star formation has been almost entirely quenched. In order to constrain this quenching mechanism, we measure $\mathrm{H} \alpha$ line emission in galaxies sorted by multiple galaxy properties as a function of redshift to what galaxy parameter best predicts quiescence. This is done for samples of the most massive, most luminous, and galaxies with the highest velocity dispersion both locally $(0.05<z<0.07$ in the SDSS ) and at high redshift $(0.7<z<1.5$ in 3D-HST). It is demonstrated through spectral stacking that velocity dispersion results in the lowest $\mathrm{H} \alpha$ line equivalent width both locally and at high redshift. The spatial distribution of the emission line flux is available from grism spectroscopy: the line flux from the high dispersion stack is centrally peaked and thus likely associated with AGN activity rather than star formation, strengthening this conclusion. Since velocity dispersion may also be the best predictor of halo mass (Wake et al. 2012), this may imply that the quenching mechanism is directly related to halo mass.
\end{abstract}

Keywords. galaxies: evolution, galaxies: high-redshift
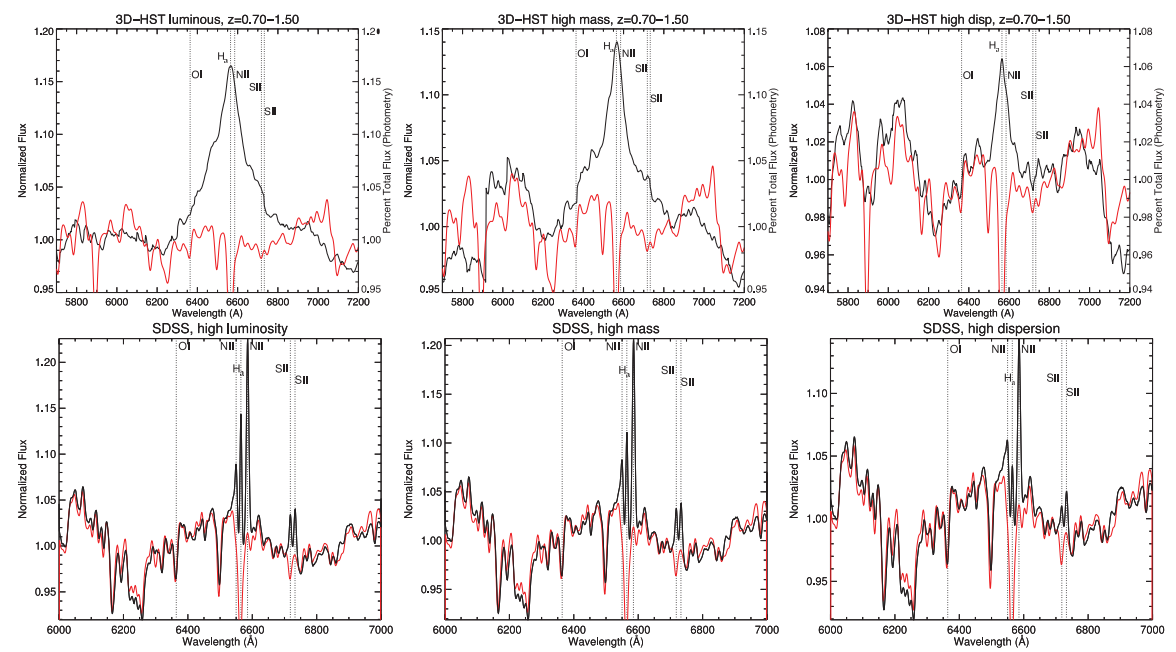

Figure 1. Stacks of SDSS spectra and 3D-HST grism spectra with stellar population synthesis models convolved to the appropriate resolution (Vazdekis et al. 2010).

\section{References}

Wake, D. A., Franx, M., \& van Dokkum, P. G. 2012, ApJ, submitted (arXiv:1201.1913)

Vazdekis, A., Sánchez-Blázquez, P., Falcón-Barroso, J., Cenarro, A. J., Beasley, M. A., Cardiel, N., Gorgas, J., \& Peletier, R. F. 2010, MNRAS, 404, 1639 